\title{
LETHAL AND MUTAGENIC EFFECTS OF ULTRAVIOLET RADIATION ON GLOMERELLA CONIDIA
}

\author{
C. L. MA R K ERT \\ Department of Zoology, University of Michigan, Ann Arbor, Michigan, U.S.A.
}

Received January 21, 1953

Is comparative studies of the mutagenic action of ionizing and ultraviolet radiation on conidia of the fungus Glomerella (5) it was evident that the shape of the mutation curve was different for the two types of radiation. The dosemutation curve for the surviving spores was essentially linear for the ionizing radiations, but with the ultraviolet radiation the mutation curve rose with increasing dose to a peak and then declined with further increases in dose. Studies on the mutagenic effects of radiation on other fungi have generally yielded the same types of curves $(1,2,3,7)$. Although various supplementary treatments of the radiated spores have been shown to alter the frequency of mutants found at any dose, the basic shape of the curve is not changed by these treatments $(10,11)$.

The types of survival curves obtained by plotting the logarithm of the survivors against dose of radiation also vary with the type of radiation. Heavily ionizing radiation results in an exponential survival curve $(8,13)$ while the less densely ionizing $x$-radiation results in a sigmoid curve; many investigators have, however, obtained exponential survival curves with $\mathrm{x}$-rays. The non-ionizing ultraviolet radiation has generally resulted in sigmoid survival curves (1), although Norman (6) obtained exponential survival curves with Neurospora microconidia after ultraviolet radiation. Ultraviolet radiation of bacteria has yielded either sigmoid or exponential survival curves depending upon the genetic makeup of the bacteria (12).

Of these various radiobiological effects perhaps the most perplexing has been the shape of the mutation curve with ultraviolet. No satisfactory explanation has yet been advanced for the decline in the mutation rate at high doses of ultraviolet (3). The present investigation was undertaken primarily to elucidate the factors involved in this peculiarity of the dose-mutation curve with ultraviolet and to relate this effect if possible to some characteristic of the survival curve. 


\section{MATERIALS AND METHODS}

Conidia of the standard type $\left(\mathrm{A}^{1} \mathrm{~B}^{1}\right)$ of the fungus Glomerella cingulata were used in this investigation. The standard type differs by gene changes at two loci from the wild type $\left(\mathrm{A}^{+} \mathrm{B}^{+}\right)$which is usually the type isolated from nature. The $\mathrm{A}^{1} \mathrm{~B}^{1}$ type produces no perithecia but when growing on appropriate media abundantly produces uninucleate haploid conidia dispersed over the mycelium (cf. Markert (5) for culture methods, etc.).

In the preparation of spore suspensions for irradiation the conidia were washed from the surface of month old cultures and filtered through cotton to remove any clumps of spores. The spores were then examined microscopically and the approximate concentration determined by counting with the aid of a hemocytometer. The density of the spore suspension was then adjusted to approximately one million per $\mathrm{ml}$. Fifteen $\mathrm{ml}$ of this suspension were then added to an open Petri dish placed on a slightly inclined rotating turntable beneath a Westinghouse sterilamp which emits over 80 per cent of its radiant energy in the region of $2537 \AA$. The distance from the spore suspension to the bottom of the ultraviolet lamp varied between 9 and 9.5 $\mathrm{cm}$. At this distance the intensity of the radiation was approximately $4770 \mathrm{ergs} / \mathrm{cm}^{2} /$ sec. The depth of the spore suspension in the Petri dish averaged about $2.4 \mathrm{~mm}$. A Glomerella conidial suspension of two million/ml absorbs less than 3 per cent of the

TABLE I

Response of sensitive and resistant Glomerella conidia to ultraviolet radiation.

\begin{tabular}{|c|c|c|c|c|c|c|c|c|}
\hline \multirow{2}{*}{$\begin{array}{l}\text { Radiation } \\
\text { dose in } \\
\text { seconds }\end{array}$} & \multirow{2}{*}{$\begin{array}{c}\text { Survivors } \\
\text { per } \\
\text { million }\end{array}$} & \multirow{2}{*}{$\begin{array}{l}\log \\
\text { survivors }\end{array}$} & \multirow{2}{*}{$\begin{array}{l}\text { number } \\
\text { of colonies } \\
\text { evaluated }\end{array}$} & \multirow{2}{*}{ Mutants } & \multirow{2}{*}{$\begin{array}{l}\text { Per cent } \\
\text { mutants }\end{array}$} & \multicolumn{3}{|c|}{$\begin{array}{l}\text { Data corrected to exclude } \\
\text { resistant spores }\end{array}$} \\
\hline & & & & & & colonies & mutants & $\begin{array}{l}\text { Per cent } \\
\text { mutants }\end{array}$ \\
\hline 0 & 1000000 & 6.0000 & 500 & 0 & 0 & 500 & 0 & 0 \\
\hline 30 & 620500 & 5.7927 & 310 & 21 & 6.8 & 310 & 21 & 6.8 \\
\hline 60 & 244600 & 5.3885 & 629 & 122 & 19.4 & 628 & 122 & 19.4 \\
\hline 90 & 38500 & 4.5855 & 642 & 233 & 36.3 & 637 & 233 & 36.5 \\
\hline 120 & 6550 & 3.8162 & 624 & 256 & 41.0 & 595 & 254 & 42.7 \\
\hline 180 & 345 & 2.5378 & 717 & 183 & 26.0 & 224 & 124 & 55.4 \\
\hline 240 & 194 & 2.2878 & 498 & 79 & 15.9 & - & - & $\ldots$ \\
\hline 300 & 153 & 2.1847 & 101 & 17 & 16.8 & - & - & - \\
\hline 360 & 134 & 2.1271 & & & & & & \\
\hline 500 & 51 & 1.7070 & & & & & & \\
\hline 600 & 22 & 1.3424 & & & & & & \\
\hline 900 & 20 & 1.3010 & & & & & & \\
\hline 1200 & 13 & 1.1139 & & & & & & \\
\hline 1500 & 8 & 0.9031 & & & & & & \\
\hline
\end{tabular}

$14770 \mathrm{ergs} / \mathrm{cm}^{2} / \mathrm{sec}$. 
incident radiation at $2537 \AA$ with a path length of one cm as determined by measurements in a Beckman spectrophotometer. This method of irradiating the spores should insure that all spores are cqually irradiated. As a check, however, several radiation experiments were performed using a rotating quartz flask to hold the spore suspension. The results from these experiments were not significantly different from those in which Petri dish containers were used.

During the course of the radiation $1 \mathrm{ml}$ aliquots were removed at intervals and plated out at appropriate dilutions in a complete agar medium. In order to minimize photoreactivation all manipulations of irradiated conidia were performed in dim light and the Petri dishes containing the plated out spores were incubated in the dark at $25^{\circ} \mathrm{C}$.

The control concentration of viable spores was determined by calculation from the dilution factor and the average number of colonies per Petri dish. The fraction of surviving spores at each dose of radiation was calculated in the same fashion and recorded as the number of spores surviving per million viable spores in the initial suspension.

In order to determine the mutation rate it was necessary to transfer all the viable colonies surviving on any selected Petri dish to agar slants in culture tubes. At four days and at six days of growth on the agar slants at $25^{\circ} \mathrm{C}$ an evaluation of all the colonies was made. Those which were visibly different from the control cultures at either time were classified as mutant (5). The percentage of mutant colonies among the survivors was then plotted against dose to obtain the mulation curve.

\section{RESULTS}

The results of these experiments are summarized in Table I and Fig. 1. In each of eleven separate experiments on the lethal effects of ultraviolet radiation on Glomerella conidia a plot of the log of the surviving spores against dose gave a distinctly sigmoid curve which leveled off to form a long plateau. The individual values (survival curve) plotted in Fig. 1 represent the average of these eleven experiments.

The points on the mutation curve represent the percentage of mutants found among the viable colonies at each dose of radiation. The data on mutation are listed in Table I. The shape of the mutation curve corresponds with that found after ultraviolet radiation of other fungus spores $(10,1)$. The curve first rises to a peak and then declines with increasing dose of radiation.

The sigmoid survival curve clearly indicates a cumulative effect of the radiation and on the assumption of a multiple hit target hypothesis would correspond to about a 7 hit curve. This type of survival curve would also be obtained as the consequence of an indirect effect of the radiation producing a toxic substance with a threshold concentration for killing the spores.

The long plateau at the end of the survival curve implies that the irradiated 
spore suspension is not homogeneous but is composed of spores with different degrees of sensitivity to the ultraviolet radiation. The shape of the curve does not critically indicate whether the heterogeneity was present at the outset or was induced by the radiation itself. However, the height of the

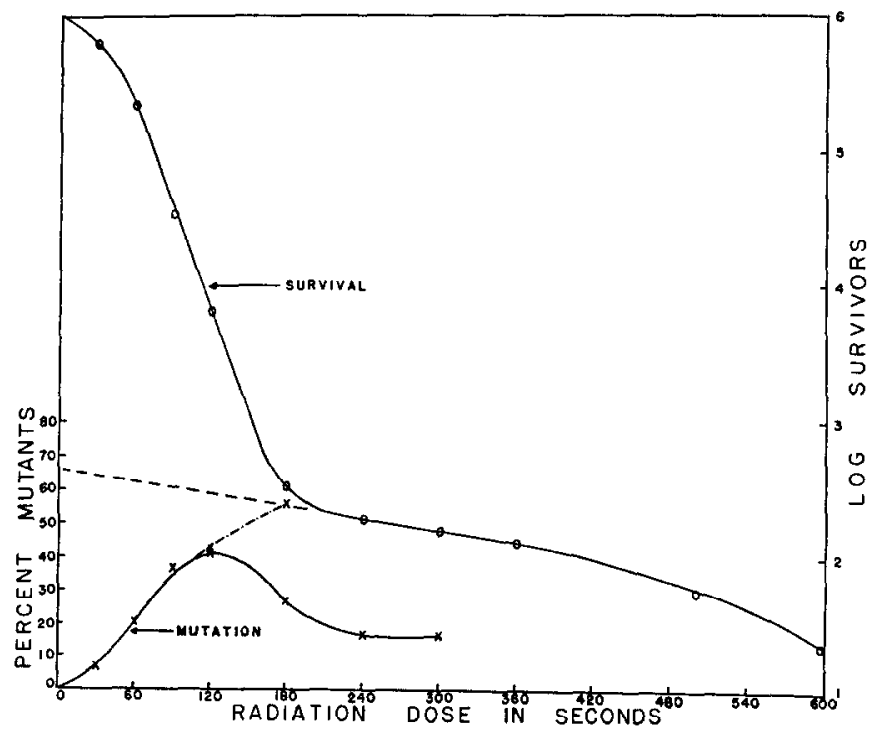

Fig. 1. Relationship of survival and mutation to dose of ultraviolet. The higher points for mutation at 120 and 180 seconds are for sensitive spores alone.

plateau above the base line--that is, the fraction of resistant spores in the suspension, varied with the age of the culture from which the spore suspension was obtained, the older cultures containing more of the resistant spores. This correlation between the number of resistant spores and the age of the spore culture leads to the conclusion that the Glomerella spore suspensions were heterogeneous before radiation. In order to insure identical responses to radiation, it was necessary to use spore cultures of exactly the sane age and environmental history.

Several experiments were performed in an effort to identify and characterize the resistant fraction of the spore population. Since a genetic basis for radiation resistance has been demonstrated in $E$. coli (12), the possibility that the resistant spores were mutants was tested. Colonies were grown from resistant spores and allowed to sporulate. The conidia were then subjected to the same radiation treatment given the conidia from the stock cultures. The survival curves describing the response of these conidia from the resistant colonies were not significantly different from the survival curves obtained with the stock conidia. These tests also exclude the possibility that the 
resistant spores might owe their resistance to a temporary diploidy produced by a precocious chromosomal division. Such a diploid spore on germination might yield two individually inviable haploid colonies which could survive, however, in symbiotic partnership. However, after sporulation such a mixed colony would be detected by the inviability of its spores. Thus the resistant spores must represent physiological or morphological variants without any genetic basis.

Although the technique of preparing the spore suspension should exclude clumps of spores, the possibility was examined that a few clumps were formed during the radiation and that these clumps provided the necessary protection to the centrally located spores to enable them to survive high doses of radiation. A microscopic examination was therefore made of the Petri dish cultures of young colonies growing from spores exposed to very high doses of radiation ( 1500 seconds). This examination failed to reveal any clumps of spores but it was immediately apparent that in every instance the germinating spores were highly pigmented. Normally Glomerella conidia appear pink in large masses but essentially colorless when viewed individually under the microscope. The pigmented resistant spores were black as shown in Fig. 2. The black pigment is presumably a melanin produced as the result of tyrosinase activity. Glomerella conidia as well as the mycelia have been shown to contain large quantities of tyrosinase (9). No explanation is at present available to account for the fact that only a very small fraction of the spore population becomes pigmented although all of the spores contain the necessary enzyme for synthesizing the melanin.

The presence of resistant pigmented spores in a normal spore population suggested an explanation for the shape of the mutation curve (Fig. 1). At increasing doses of radiation the surviving spores would be composed of increasing proportions of the resistant variety (resistant both to the mutagenic and lethal action of the ultraviolet) and thus the proportion of mulable spores (and of mutants) would decline at increasing doses. By extrapolating the. plateau portion of the survival curve back to zero dose an estimate of the number of resistant spores per million spores of the initial population may be obtained. In spore cultures of the age (one month) used in these experiments the resistant spores number about 470 per million spores.

The mutation frequencies used in plotting the mutation curve (Fig. 1) were based almost entirely upon mutations of sensitive spores. In order to describe accurately the frequency of mutation among sensitive spores it is necessary to make a correction for the presence of resistant spores in the initial spore population. The resistant spores surviving at any given dose 
may be estimated from the appropriate point on the extrapolated portion of the plateau of the survival curve (dashed line in Fig. 1). Although the extrapolated portion of the curve should be sigmoid rather than linear as drawn, the deviation from linearity at doses of 120 and 180 seconds would be too small to affect the data significantly. After 240 seconds of radiation essentially only resistant spores survive. The low percentage of mutation at this dose indicates that even the resistant spores are eventually mutated or killed by the ultraviolel radiation.

Thus in calculating the mutation rate for sensitive spores alone it is necessary to subtract the surviving resistant spores from the number of colonies evaluated and also to subtract the mutants contributed by the resistant colonies from the total mutants found. The mutants from resistant colonies were calculated on the assumption of a linear dose-mutation relation for these resistant spores up to 240 seconds at which point the mutation rate was observed to be approximately 16 per cent. The corrected mutation frequencies for sensitive spores are listed in Table I and are depicted by the dotted mutation curve in Fig. 1. It is apparent that the mutation rate for sensitive spores alone does not decline with increasing doses of radiation.

The existence of melanin pigment in a few of the spores from month old cultures serves to separate the spore population into two distinct and fairly homogeneous groups each with its own distinct sensitivity both to the mutagenic and to the lethal effects of the ultraviolet radiation. The relationship between the mutagenic and lethal effects of the radiation is shown by the fact that the ratio of the slopes of the mutation curves (about $6: 1$ ) for the two types of spores corresponds closely to the ratio of their relative resistance to the lethal effects of the radiation.

\section{DISCUSSION}

The survival and mutation curves obtained in this investigation with spores of Glomerella are very similar to those obtained by Hollaender and Emmons (1) with ultraviolet irradiated spores of the fungus Trichophyton mentagrophytes. These investigators and others $(10)$ bave generally attributed the decline in the mutation rate at high doses of ultraviolet to an increased sensitivity of mutated spores to the lethal action of the radiation. Although such an hypothesis is consistent with the previously known facts, it would appear from the Glomerella example that a more probable explanation must be based upon the presence of resistance-conferring morphological or physiological characterstics in a small fraction of the spores in the initial population. 

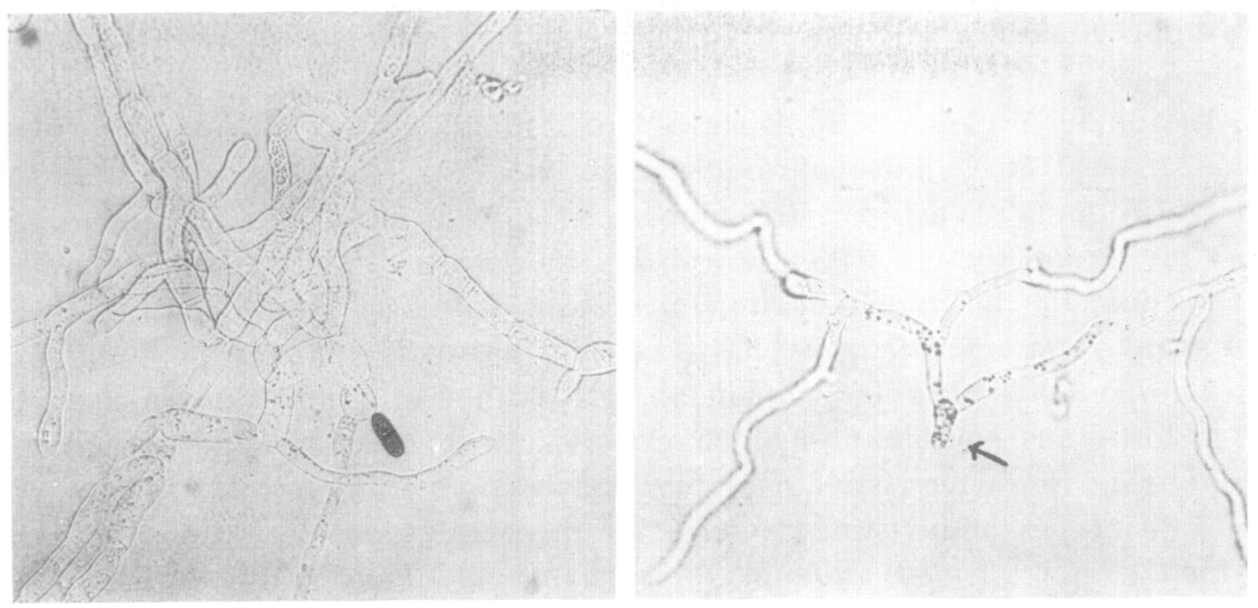

Fig. 2. (Jeft) Resistant pigmented spore germinating after exposure to 1500 sees of ultraviolet radiation. (Right) Unirradiated colorless sensitive spore germinating.

So far as susceptibility of mutants to ultraviolet is concerned there is direct evidence that numerous mutants of Glomerella do not differ from standard type in their response to ultraviolet. In experiments on ultraviolet induced back mutations several nutritional and morphological mutants were exposed to ultraviolet at varying doses. Their survival curves were not significantly different from those of the standard type. Unfortunately these experiments were done before the melanin basis for ultraviolet resistance was known and no effort was made to pick mutants with greatly different amounts of tyrosinase. Mutants differing in tyrosinase content might well show corresponding variations in the proportion of resistant pigmented spores produced in a spore culture.

In quantitative irradiation experiments with the fungus Neurospora crassa, Hollaender et al. (3) also found the typical rise and decline of the mutation curve with increasing doses of ultraviolet. They suggested that the initial spore suspension might be heterogeneous in respect to the amount of the radiation absorbed by each spore because of variations in the ultraviolet absorption by the spore wall and cytoplasm at different ages of development. Efforts by them to test this hypothesis on spores of different ages yielded inconclusive results. Although this hypothesis was not clearly verified for Neurospora conidia, it is evidently the correct explanation for the bchavior of Glomerella conidia, in which protective melanization is partly a function of age. Since Neurospora mycelia contain tyrosinase (4) it may yet be found 
that the conidia also contain tyrosinase and variable amounts of melanin pigment.

In another organism-the B strain of $E$. coli-Witkin (12) has shown that the survival curve after ultraviolet irradiation decreases in slope at high doses. Although genetically resistant bacteria occur among large populations of this strain, these genetically resistant bacteria are not numerous enough to account for the change in slope of the survival curve. Some non-inherited protective characteristic must be present in part of the bacterial population.

The fact that melanin pigment absorbs both visible and ultraviolet light is a fortuitous combination of characteristics which makes the resistanceconferring character visible under the microscope. However, there may well be other types of invisible chemical or physical changes in fungus spores which confer varying amounts of resistance to ultraviolet radiation. The melanized Glomerella conidia are only one example that such mechanisms do exist.

\section{SUMMARY}

1) The survival curves of ultraviolet irradiated Glomerella conidia have a sigmoid shape. The sigmoid survival curve may be described as a 7 hit curve (approximately) or as indicating radiation-produced toxic substances with a threshold for lethal action.

2) At high doses of radiation the survival curve levels off to form a long plateau. 'This plateau in the curve indicates the presence of a small proportion of resistant spores among the sensitive population. The resistant spores may be observed under the microscope and are characterized by the presence of melanin pigment in the spore in contrast to the great majority of Glomerella conidia which appear colorless. The proportion of resistant spores increases with increasing age of the spore culture.

3) With increasing dose of ultraviolet radiation the mutation curve rises to a peak and then declines at higher doses of radiation. This decline in the frequency of mutants among the surviving spores is due to an increasing proportion of resistant pigmented spores among the surviving population at high doses. The mutation curve for sensitive spores alone does not decline at the higher doses of radiation.

The author wishes to acknowledge the careful assistance of Miss Bonnie Gobble and Miss Patricia A. Caughey. This investigation was aided by grants from the Atomic Energy Commission under contract No. AT (11-1) -70 project No. 4 with the University of Michigan. 


\section{REFERENCES}

1. Hollaender, A., and Emmons, C. W., Cold Spring Harbor Symposia Quant. Biol., 9, 179 (1941).

2. Hollaender, A., Raper, K. B., and Coghill, R. D., Am. J. Bot., 32, 160 (1945).

3. Hollaender, A., Sansome, E. R., Zimmer, E., and Demerec, M., ibid, 32, 226 (1945).

4. Honowitz, N. H., and Sines, S. C., J. Biol. Chem., 197, 513 (1952).

5. Markert, C. L., Genetics, 37, 339 (1952).

6. Norman, A., Exptl. Cell Research, 2, 454 (1951).

7. Sansome, E. R., Demerec, M., and Hollaender, A., Am. J. Bot., 32, 218 (1945).

8. Stapleton, G. E., Hollaender, A., and Martix, F. L., J. Cellular Comp. Physiol., 39, Suppl. 1, 87 (1952).

9. Sussman, A. S., and Markert, C. L., Arch. Biochem. Biophys. (in press).

10. Swanson, C. P., J. Cellular Comp. Physiol., 39, Suppl. 1, 27 (1952).

11. Swanson, C. P., Ilollaender, A., and Katfmaxn, B. N., Genetics, 33, 429 (1948).

12. Witkin, E. M., Genetics, 32, 221 (1947).

13. Zirkle, R. E., Marchbank, D. F., and Kuck, K. D., J. Cellular Comp. Physiol., 39, 75 (1952). 